\title{
Variable-Temperature Noise Characterization of N-MOSFETs Using an In-Situ Broadband Amplifier
}

\author{
Kenji Ohmori, Member, IEEE, and Shuhei Amakawa, Member, IEEE
}

\begin{abstract}
Characterization of broadband noise of MOSFETs from room temperature down to $120 \mathrm{~K}$ in fine temperature steps is presented. A MOSFET is mounted on a reusable printed circuit board vehicle with a built-in low-noise amplifier, and the vehicle is loaded into a cryogenic chamber. The vehicle allows noise measurement in the frequency range from $50 \mathrm{kHz}$ to $100 \mathrm{MHz}$. At low frequencies, it enables extraction of activation energies associated with electron trapping sites. At high frequencies, as has been suggested by noise figure measurements, the white noise of MOSFETs is shown to be dominated by the shot noise, which has much weaker temperature dependence than the thermal noise. The shot noise will a problematic noise source in broadband RF CMOS circuits operating at cryogenic temperatures.
\end{abstract}

Index Terms-cryogenic CMOS, white noise, 1/f noise, lowfrequency noise spectroscopy, device modeling, quantum computing

\section{INTRODUCTION}

Low temperature environment enhances performance of CMOS devices through the improvement of the carrier mobility, subthreshold slope, $\mathrm{Cu}$ wire resistance, and thermal noise [1-3]. Applications of "cold" CMOS range from high performance computing to space exploration and low temperature physics. Quantum computing is one of the challenging technologies that require that the power of CMOS circuits be made available at cryogenic temperatures. To maintain the coherence time of quantum bits (qubits), such as superconductive qubits [4-6] and semiconductor spin qubits [7], a quantum processor comprising several to several tens of qubits is put in a sub-decikelvin cryogenic chamber. At present, peripheral circuits for controlling the quantum processor typically operate at room temperature and are connected to the quantum processor via long RF cables. However, as the number of qubits increases exponentially in the near future, error correction needs to be implemented for maintaining the computation fidelity over the coherence time of qubits. Then, the control latency will be critical for continuous operation of such a quantum processor [8-11]. Efforts have been made to move low-power RF CMOS controllers into a few-kelvin chamber that encloses the sub-decikelvin chamber [10].

For the design of high-performance cryogenic RF CMOS circuits for whatever purposes, MOSFET compact models valid down to cryogenic temperatures are necessary [12-13]. At cryogenic temperatures, many performance parameters including the saturation value of the drain current $\left(I_{\mathrm{d}}\right)$ and electron mobility improve and, in addition, peculiar phenomena, such as bandgap widening, carrier freeze-out, and self-biasing, appear [14]. Therefore, continuous characterization of MOSFETs at varying temperatures starting from room temperature down to cryogenic temperatures is important for developing physics-based predictive device models. In particular, wideband noise modeling is essential for the development of low-noise circuits.

MOSFETs are known to exhibit flicker noise at low frequencies and white noise at high frequencies. Physical origins of flicker noise and white noise are different, and their power spectral densities are independent of each other. White noise can be further classified into thermal noise and shot noise. These are not completely independent of each other and become indistinguishable under a zero drain bias $[15,16]$. When a nonzero drain bias is applied, the shot noise becomes dominant $[17,18]$. White noise from a local oscillator have recently been shown to adversely affect the operation of wideband millimeter-wave circuits $[19,20]$.

The noise figure (NF) measurement is typically used to characterize the white noise of MOSFETs [21,22]. The most commonly used Y-factor method of NF measurement requires a hot noise source. In cryogenic environments, this method is not straightforwardly applicable because noise sources are not commercially available [23]. Therefore, either the cold-source method or the cold-attenuator method is employed, in which several cooling cycles per characterizing a single device under test (DUT) are required for complicated calibration [24]. On the other hand, the maximum measurable frequency of lowfrequency noise measurement systems is usually too low to cover white noise due to parasitic capacitance inherent in such systems.

A proof-of-concept noise probe for on-wafer broadband noise characterization was demonstrated at room temperature, achieving noise measurement from $100 \mathrm{kHz}$ up to $800 \mathrm{MHz}[25,26]$. This was made possible by reducing the parasitic capacitance by placing a broadband low-noise amplifier (LNA) in the probe itself. At present, an improved version of the noise probe is commercially available [27], details of which were presented in [18]. In this study, we apply the same principle to the development of an in-situ system for variable-temperature broadband noise measurements down to $120 \mathrm{~K}[28]$. 
Low-frequency noise from a very small MOSFET can reveal individual trapping/detrapping of a single electron to/from a trapping site. Using this capability, low-frequency noise measurement is commonly employed to characterize the quality of gate dielectrics [29]. In this paper, we will also demonstrate low-frequency noise spectroscopy (LFNS) [30-33], in which, by characterizing temperature-dependent noise intensities of a MOSFET, the activation energy related to electron trapping is extracted.

We will also look at the temperature dependence of white noise, especially shot noise, and the suppression of the latter compared to its theoretical maximum value (full shot noise), through the use of the so-called Fano factor or the shot-noise suppression factor.

\section{EXPERIMENTAL SETUP AND MEASUREMENT}

We developed a reusable printed circuit board (PCB) vehicle for cryogenic measurements, on which a DUT is mounted. Also on the PCB vehicle is a broadband LNA for in-situ sensing of noise from the DUT. The noise generated by the broadband LNA can be calibrated out by pre-characterizing the LNA by extensive measurements [18]. We applied this calibration approach to variable-temperature measurements so that the PCB vehicle is calibrated at every kelvin over the temperature range from 300 to $120 \mathrm{~K}$. Note that once the variabletemperature calibration of a vehicle is completed through several cooling cycles, measurements of $\mathrm{dc}$ and noise characteristics can be conducted in a single cooling cycle.

A DUT is mounted on the vehicle right next to the LNA. The configuration of electrical instruments is similar to that in [18], except that the PCB vehicle is put in a cryogenic helium chamber as schematically shown in Fig. 1. We employed a semiconductor device analyzer (B1500A, Agilent Technologies), equipped with four high-resolution sourcemeasure units (HR-SMUs), for biasing the DUT, and a spectrum analyzer (N9030A, Agilent Technologies) for reading the output from the LNA. The noise generated by the spectrum analyzer is accounted for, in effect, by turning on its Noise Floor Extension option [34]. Custom-built low-pass filters were used to filter out noise from the HR-SMUs.

We characterized N-MOSFETs from a few different semiconductor foundries. Three types of MOSFETs, denoted by $\mathrm{A} / \mathrm{B} / \mathrm{C}$, were measured. The gate lengths $(L)$ of type-A/B

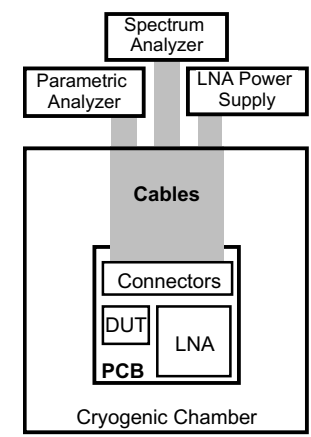

Fig. 1. Schematic of a cryogenic system with a PCB vehicle for wideband noise measurement.
MOSFETs were $240 \mathrm{~nm}$, and that of type-C was $120 \mathrm{~nm}$. Each of the DUTs had 4 contact pads (gate, drain, source, and substrate electrodes) and was mounted on a PCB vehicle by flip-chip or wire bonding for stable measurements at different temperatures. If one uses a cryogenic probe station, the probe tips shrink in length as $T$ decreases, which makes stable continuous variable- $T$ measurement very difficult. Therefore, it is beneficial also in this regard to bond a DUT next to an LNA.

DUT temperatures were measured using a silicon diode sensor attached next to the DUT on the PCB vehicle. The temperature ranges examined are from 5 to $300 \mathrm{~K}$ for dc $I-V$ measurements and 120 to $300 \mathrm{~K}$ for noise measurements. The lowest temperature of $120 \mathrm{~K}$ of the noise measurement was dictated by the carrier freeze-out of bipolar transistors in the LNA.

\section{VARIABLE-TEMPERATURE CHARACTERIZATION}

\section{A. Dc Characteristics}

Figure 2(a) shows $I_{\mathrm{d}}-V_{\mathrm{g}}$ curves at $T=5$ to $300 \mathrm{~K}$ for a typeA DUT under a drain-source voltage, $V_{\mathrm{d}}$, of $0.05 \mathrm{~V}$. The $I_{\mathrm{d}}-V_{\mathrm{g}}$ measurement was carried out at every $1-5 \mathrm{~K}$ step. At low temperatures, $I_{\mathrm{d}}$ decreases in the subthreshold region and increases in the strong inversion region. The change in the transconductance $g_{\mathrm{m}}$ is shown in Fig. 2(b). The dependence of the threshold voltage $V_{\text {th }}$ on $T$ is plotted in Fig. 3(a). $V_{\text {th }}$ was extracted by drawing a tangential line on an $I_{\mathrm{d}}-V_{\mathrm{g}}$ curve at the point where $g_{\mathrm{m}}$ assumes a maximum value. $V_{\text {th }}$ shifts from $0.74 \mathrm{~V}$ at $300 \mathrm{~K}$ to $0.94 \mathrm{~V}$ at $5 \mathrm{~K}$. The electron current density is expressed as

$J_{\mathrm{n}}=q n \mu_{\mathrm{n}} \mathcal{E}+q D_{\mathrm{n}} \nabla n$,
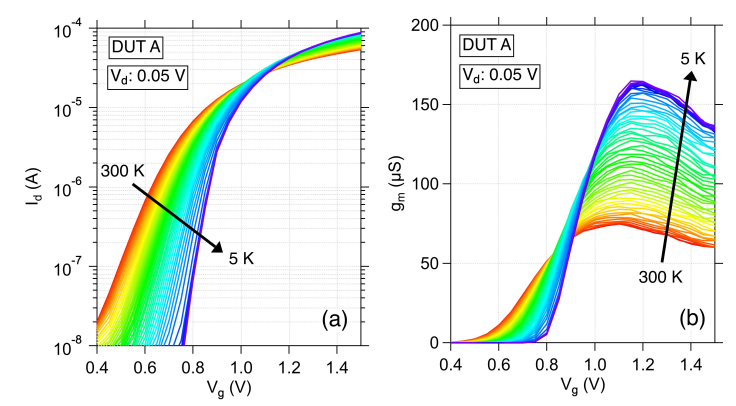

Fig. 2. (a) Typical MOSFET $I_{\mathrm{d}}-V_{\mathrm{g}}$ characteristics and (b) transconductance $g_{\mathrm{m}}$ at temperatures ranging from 5 to $300 \mathrm{~K}$.
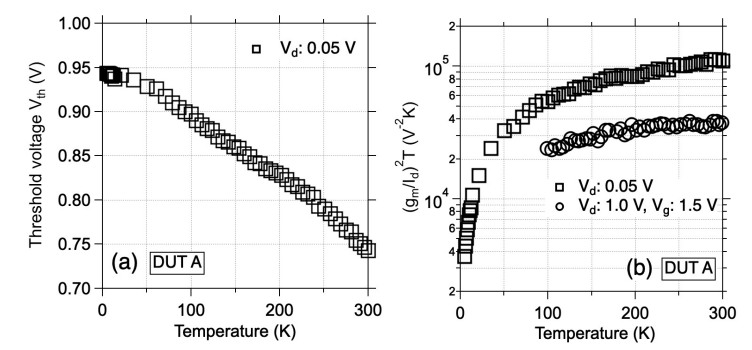

Fig. 3. (a) Threshold voltage $V_{\text {th }}$ versus $T$ for $V_{\mathrm{d}}=0.05 \mathrm{~V}$. (b) $\left(g_{\mathrm{m}} / I_{\mathrm{d}}\right)^{2} T$ versus $T$ for $V_{\mathrm{d}}=0.05$ and $1.0 \mathrm{~V}$. 
where $n$ is the concentration of electrons in the inversion layer, $\mu_{\mathrm{n}}$ is the electron mobility, $\mathcal{E}$ is the electric field, and $D_{\mathrm{n}}$ is the diffusion coefficient, which is proportional to $T$. The diffusioncurrent component of $I_{\mathrm{d}}$ decreases as the temperature becomes lower, resulting in higher $V_{\text {th }}$ [Fig. 3(a)] and steeper subthreshold slope [Fig. 2(a)]. Figure 3(b) shows $\left(g_{\mathrm{m}} / I_{\mathrm{d}}\right)^{2} T$ versus $T$ for $V_{\mathrm{d}}=0.05 \mathrm{~V}$ and $1.0 \mathrm{~V}$. The ratio $g_{\mathrm{m}} / I_{\mathrm{d}}$ is the normalized (per-unit-drain-current) transconductance, often examined in relation to surface potential fluctuations $[35,36]$. The peak $g_{\mathrm{m}}$ values in Fig. 2(a) and the corresponding $I_{\mathrm{d}}$ were used to estimate $\left(g_{\mathrm{m}} / I_{\mathrm{d}}\right)^{2} T$ for $V_{\mathrm{d}}=0.05 \mathrm{~V}$ (open squares) in Fig. 3(b), while for $V_{\mathrm{d}}=1.0 \mathrm{~V}$ (open circles), $g_{\mathrm{m}}$ and $I_{\mathrm{d}}$ values at $V_{\mathrm{g}}=1.5 \mathrm{~V}$ were used because $g_{\mathrm{m}}-V_{\mathrm{g}}$ curves (not shown) exhibited monotonic increase at $V_{\mathrm{d}}<1.5 \mathrm{~V}$. $\left(g_{\mathrm{m}} / I_{d}\right)^{2} T$ is to be considered later in connection with the dependence of normalized noise intensity on $T$.

Figure 4 shows $I_{\mathrm{d}}-V_{\mathrm{d}}$ curves at $T=122$ to $300 \mathrm{~K}$ for a typeA DUT under (a) $V_{\mathrm{g}}=0.9$ and (b) $1.5 \mathrm{~V} . I_{\mathrm{d}}$ in Fig. 4(a) decreases as $T$ decreases, whereas $I_{\mathrm{d}}$ in Fig. 4(b) increases, consistent with the behavior observed in Fig. 2(a). The dependence of $I_{\mathrm{d}}$ on $T$ under a given bias condition is affected
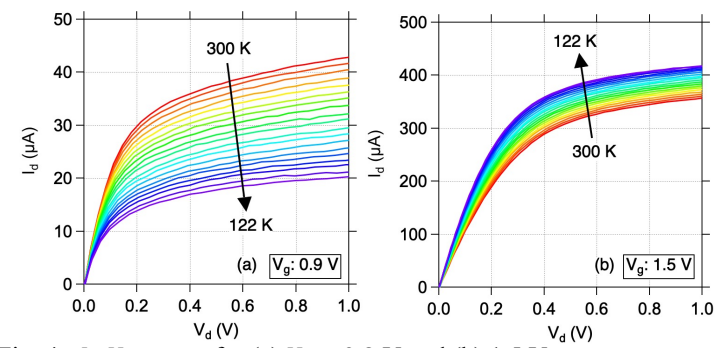

Fig. 4. $I_{\mathrm{d}}-V_{\mathrm{d}}$ curves for (a) $V_{\mathrm{g}}=0.9 \mathrm{~V}$ and (b) $1.5 \mathrm{~V}$.
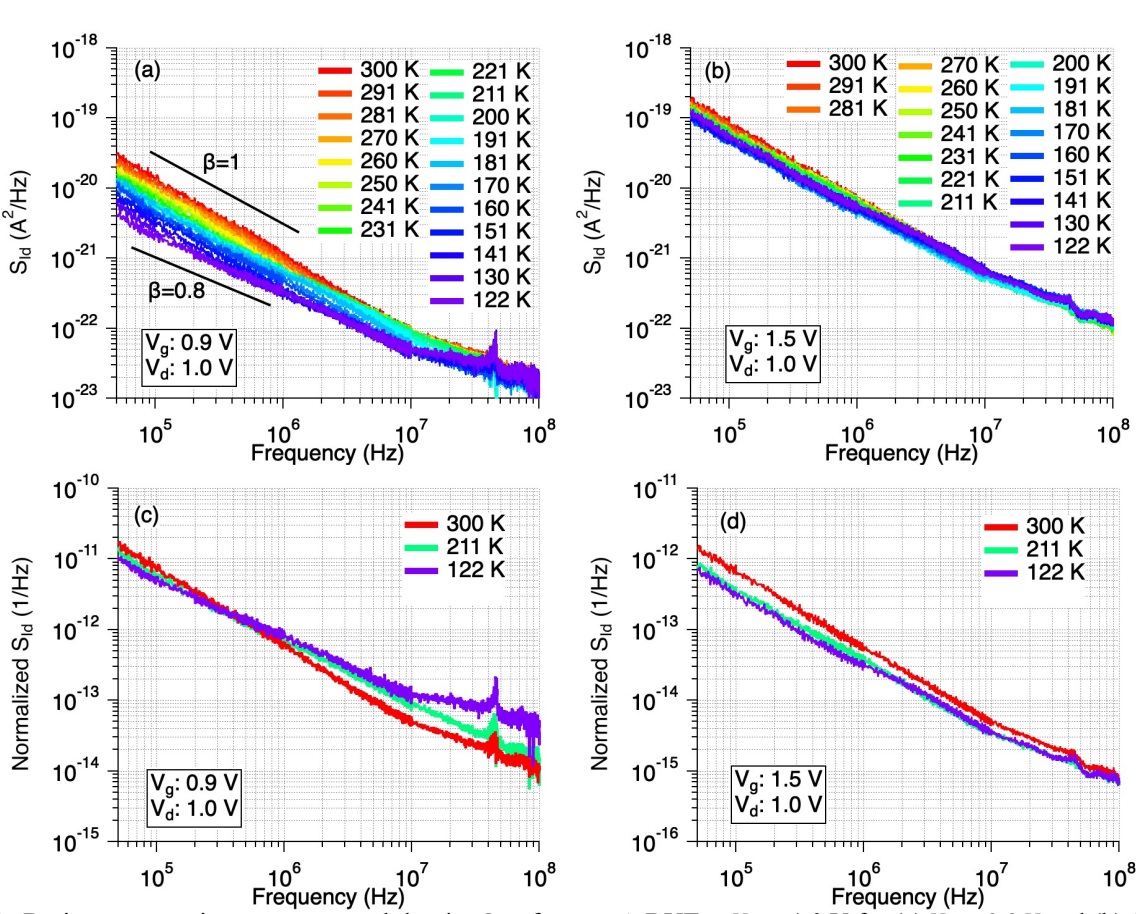

by many factors including the process technology node, the structure of the MOSFET, and the impurity concentration [2]. Therefore, continuous variable-temperature characterization is necessary for physics -based predictive device modeling.

\section{B. I/f Noise in the Saturation Region}

Before focusing on white noise, we demonstrate a measurement result using the vehicle in the low-frequency, flicker-noise region. Changes in drain-current noise power spectral density $S_{\text {Id }}$ for a type-A DUT are shown in Fig. 5(a,b). The bias conditions are (a) $V_{\mathrm{g}}=0.9$ and (b) $1.5 \mathrm{~V}$ with $V_{\mathrm{d}}=$ $1.0 \mathrm{~V}$ in both cases. The corresponding $I_{\mathrm{d}}$ values can be found in Fig. 4. $S_{\text {Id }}$ normalized by $I_{\mathrm{d}}^{2}$ for selected temperatures is shown in Fig. 5(c,d). $1 / f^{\beta}$ frequency dependence is observed with $\beta$ gradually decreasing in the high-frequency region above $10 \mathrm{MHz}$, where $-\beta$ is the slope of the noise power spectrum on a $\log -\log$ plot. The $\beta$ value for $V_{\mathrm{g}}=0.9 \mathrm{~V}$ extracted in the frequency range from $100 \mathrm{kHz}$ to $1 \mathrm{MHz}$ decreases from 1.07 at $300 \mathrm{~K}$ to 0.77 at $122 \mathrm{~K}$. As shown in Fig. 3(a), $V_{\text {th }}$ is a decreasing function of temperature. We used the gate-source voltage, $V_{\mathrm{g}}$, as biasing points in variable-temperature noise measurements. Under the condition of $V_{\mathrm{g}}=0.9 \mathrm{~V}$, the channel is in strong inversion, and the gate overdrive voltage decreases as $T$ decreases. The change in $\beta$ for $V_{\mathrm{g}}=0.9 \mathrm{~V}$ in Fig. 5(a) could be related to a depth-dependent trap profile in the oxide layer [37]. Note that the magnitudes of normalized $S_{\text {Id }}$ for 122 and $300 \mathrm{~K}$ intersect in Fig. 5(c) and the inequality is reversed at $400 \mathrm{kHz}$. Studies on low frequency noise are typically conducted in a frequency range from $1 \mathrm{~Hz}$ to $10 \mathrm{kHz}$, or at most $100 \mathrm{kHz}$. It does appear important that the measurement frequency range is significantly extended when trying to

Fig. 5. Drain-current noise power spectral density $S_{\text {Id }}$ of a type-A DUT at $V_{\mathrm{d}}=1.0 \mathrm{~V}$ for (a) $V_{\mathrm{g}}=0.9 \mathrm{~V}$ and (b) $1.5 \mathrm{~V}$. $S_{\text {Id }}$ normalized by $I_{\mathrm{d}}^{2}$ for (c) $V_{\mathrm{g}}=0.9 \mathrm{~V}$ and (d) $1.5 \mathrm{~V}$. 
develop predictive device noise models valid in various operating conditions, including low temperatures.

On the other hand, the values of $\beta$ do not change very much when $V_{\mathrm{g}}=1.5 \mathrm{~V}$ and $V_{\mathrm{d}}=1.0 \mathrm{~V}$ as shown in Fig. 5(b,d). Although $I_{\mathrm{d}}$ increases as $T$ decreases [Fig. 4(b)], $S_{\text {Id }}$ (and obviously, normalized $S_{\text {Id }}$ ) decrease. The relationship between the normalized $S_{\mathrm{Id}}$ and $\left(g_{\mathrm{m}} / I_{d}\right)^{2}$ is expressed as [36]

$$
\frac{S_{\mathrm{Id}}}{I_{\mathrm{d}}^{2}}=\left(\frac{g_{\mathrm{m}}}{I_{\mathrm{d}}}\right)^{2} \frac{q^{2} \rho \lambda k_{\mathrm{B}} T}{C_{\mathrm{ox}} W L f}
$$

where $\rho$ is the density of the McWhorter states in the oxide, $\lambda$ is the electron wave penetration depth in the oxide, $k_{\mathrm{B}}$ is the Boltzmann constant, $C_{\mathrm{ox}}$ is the capacitance of the oxide per unit area, and $W$ is the gate width. $\left(g_{\mathrm{m}} / I_{\mathrm{d}}\right)^{2} T$, shown in Fig. 3(b), for the same bias condition also decreases as $T$ decreases. This is consistent with the decrease in normalized $S_{\text {Id }}$ in the lowfrequency region in Fig. 5(d).

\section{Low-Frequency Noise Spectroscopy (LFNS)}

Next, we conduct an analysis of the physical origin of the observed low-frequency noise. Trapping and detrapping of electrons, typically at the interface between the gate dielectric film and the channel of a MOSFET, result in random telegraph noise (RTN) in the time domain [38]. This is often observed as waveforms exhibiting two discrete levels with a certain time constant $\tau$ of transition between the two levels, corresponding to capture and emission of an electron. In the frequency domain, RTN appears as a Lorentzian-shaped hump at a characteristic frequency $f_{\tau} \approx(2 \pi \tau)^{-1}$ [38]. Figure 6 shows changes in draincurrent noise power spectral density $S_{\text {Id }}$ for a type-B DUT at different temperatures, exhibiting the Lorentzian behavior with temperature-dependent $f_{\tau}$. Since the time constant is a function of the activation energy and $T$, the frequency $f_{\tau}$ shifts to higher frequencies as $T$ rises. Figure 7(a) shows $S_{\text {Id }}$ versus temperature extracted at frequencies ranging from $50 \mathrm{kHz}$ to $5 \mathrm{MHz}$. Two sets of peaks, labeled "Peak 1" and "Peak 2," were observed. The peak temperatures depend on the frequency. For an electron trap in a MOSFET, the relationship between $T$ and $f$ is expressed as [30]

$$
\ln \left(\frac{T^{2}}{f}\right)=\frac{E_{\mathrm{c}}-E_{\mathrm{t}}}{k_{\mathrm{B}} T}+C
$$

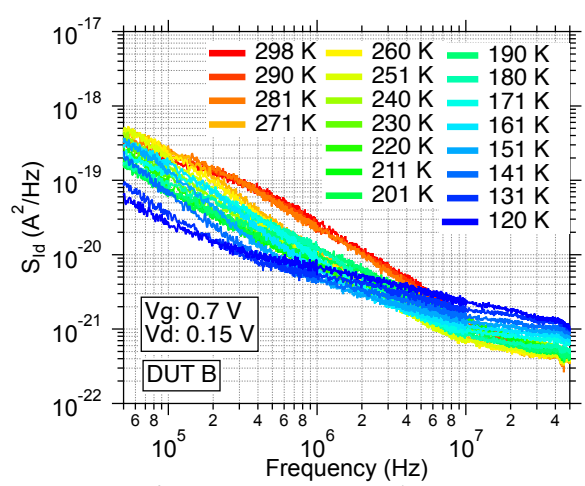

Fig. 6. $S_{\text {Id }}$ spectra of a type-B DUT at various temperatures, showing wavy curves resulting from RTN. where $E_{\mathrm{c}}$ is the bottom energy of the conduction band, $E_{\mathrm{t}}$ is the trap level, and $C$ is a constant that includes the capture cross section of the trap and the effective mass of electrons. This allows us to use the Arrhenius method of estimating the activation energy $E_{\mathrm{c}}-E_{\mathrm{t}}$. Figure 7(b) shows an Arrhenius plot for the peak temperatures and frequencies extracted from Fig. 7(a). The values of $E_{\mathrm{c}}-E_{\mathrm{t}}$ were found to be 0.21 and $0.27 \mathrm{eV}$ for "Peak 1" and "Peak 2," respectively. Identification of trap species, for example, hydrogen, interstitial carbon, and interstitial boron, was reported [32], in which the activation energies ranged from 0.10 to $0.44 \mathrm{eV}$. To do the same here, further investigation using more DUTs will be required.

In quantum computing, a major cause of decoherence is noise, which is often generated by defects and traps in close proximity to qubits [39]. LFNS is applicable to systems in which current or voltage is affected by trapping phenomena [31]. LFNS could also be employed for studies of improving the coherence time of qubits.
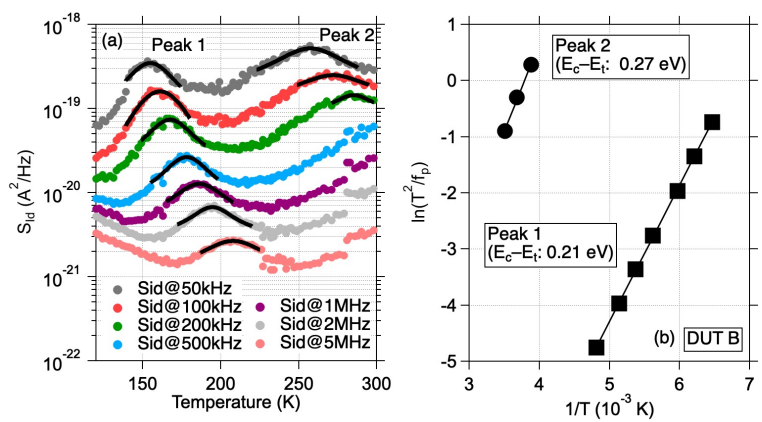

Fig. 7. (a) Low-frequency noise spectroscopy for a type-B DUT. Two sets peaks were observed. (b) Arrhenius plots for extracting activation energies.

\section{Pure Thermal Noise at Zero Drain Voltage}

What is unique about using the vehicle with the built-in broadband LNA is the capability to characterize white noise, depending on the bias condition. Figure 8 shows $S_{\text {Id }}$ versus frequency under zero-drain-bias $\left(V_{\mathrm{d}}=0 \mathrm{~V}\right)$ conditions with $V_{\mathrm{g}}$ from 0.8 to $1.6 \mathrm{~V}$ at (a) $T=300$ and (b) $121 \mathrm{~K}$. The frequency range is from $50 \mathrm{kHz}$ to $100 \mathrm{MHz}$. Note that the maximum measurable frequency in commercially available lowfrequency noise systems is a few tens of $\mathrm{MHz}$ at the highest. The observed white noise in Fig. 8 can be regarded either as thermal noise, given by $4 k_{\mathrm{B}} T / R_{\mathrm{dif}}$, where $R_{\mathrm{dif}}=\partial V_{\mathrm{d}} / \partial I_{\mathrm{d}}$, or as shot noise [40]. As $V_{\mathrm{g}}$ increases, $R_{\text {dif }}$ decreases, resulting in higher white noise levels.

Noise measurements were conducted continuously from 120 to $300 \mathrm{~K}$. In Fig. 9, temperature dependence of extracted $S_{\text {Id }}$ values at $10 \mathrm{MHz}$ for (a) $V_{\mathrm{g}}=1.0 \mathrm{~V}$ and (b) $V_{\mathrm{g}}$ ranging from 0.8 to $1.6 \mathrm{~V}$ are plotted as a function of the theoretical thermal noise intensity $S_{\text {th }}\left(=4 k_{\mathrm{B}} T / R_{\mathrm{dif}}\right)$. As the temperature becomes low, $S_{\text {th }}$ and hence $S_{\text {Id }}$ decrease. We observed a good linear relationship between $S_{\text {th }}$ and $S_{\text {Id }}$ with a slope of unity. The range of the linear relationship in Fig. 9 (b) is from $4 \times 10^{-25}$ to $7 \times 10^{-23} \mathrm{~A}^{2} / \mathrm{Hz}$, which corresponds to $R_{\text {dif }}$ values from $23 \mathrm{k} \Omega$ to $188 \Omega$. 

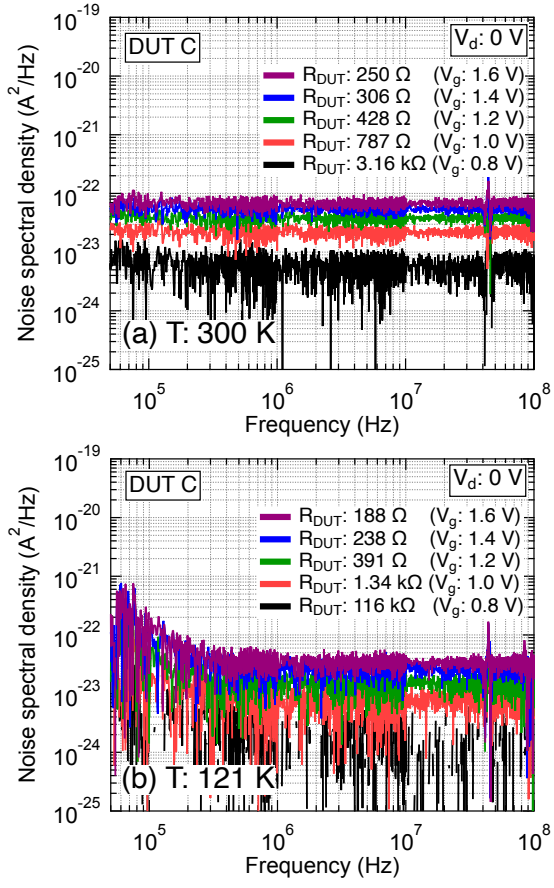

Fig. 8. Noise power spectral density $S_{\mathrm{Id}}$ of a type-C DUT obtained at $V_{\mathrm{d}}=0 \mathrm{~V}$ and at (a) $T=300$ and (b) $121 \mathrm{~K}$. Under the same bias condition, the white noise level at $T=121 \mathrm{~K}$ is lower than that at $T=$ $300 \mathrm{~K}$.

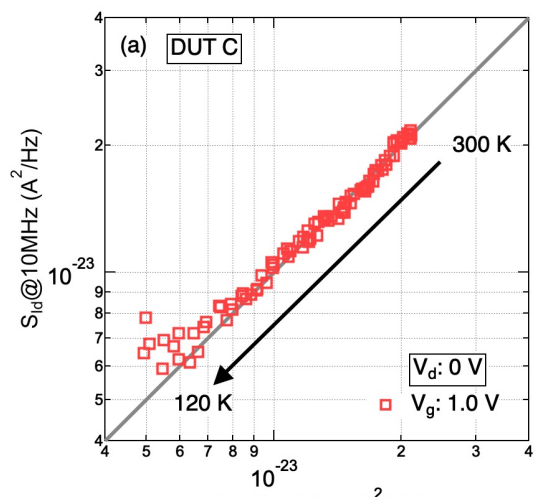

$\mathrm{S}_{\mathrm{th}}, 4 \mathrm{k}_{\mathrm{B}} \mathrm{T} / \mathrm{R}_{\mathrm{dif}}\left(\mathrm{A}^{2} / \mathrm{Hz}\right)$

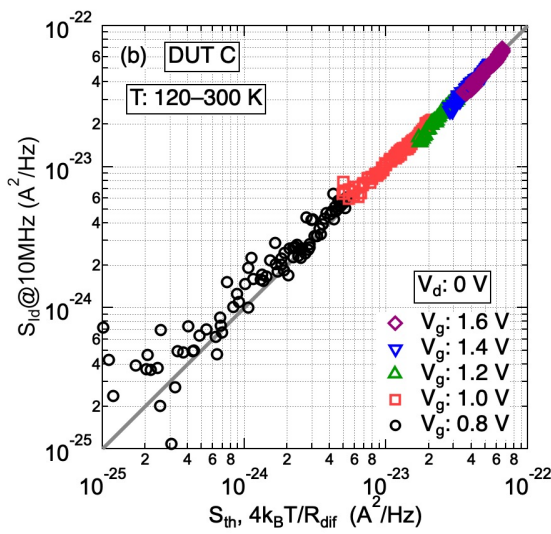

Fig. 9. (a) Change in $S_{\text {Id }}$ of a type-C DUT extracted at $10 \mathrm{MHz}$ versus the theoretical thermal noise $\left(4 k_{\mathrm{B}} T / R_{\mathrm{dif}}\right)$ with $T$ ranging from 300 to $120 \mathrm{~K}$. The gate voltages $V_{\mathrm{g}}$ are (a) $1.0 \mathrm{~V}$ and (b) $0.8,1.0,1.2,1.4,1.6 \mathrm{~V}$.

\section{E. Shot-Noise Evaluation in the Linear Region}

When a nonzero $V_{\mathrm{d}}$ is applied, $I_{\mathrm{d}}$ starts to increase and shot noise component that cannot be regarded as thermal noise emerges. In order to characterize white noise that consists of thermal and shot noise, noise measurement was conducted in the linear region of MOSFET operation using the type-A DUT, which showed the lowest levels of flicker noise. Figure 10 shows $S_{\text {Id }}$ for $V_{\mathrm{g}}=1.5 \mathrm{~V}$ and $V_{\mathrm{d}}=0,0.02,0.04,0.06$, and $0.08 \mathrm{~V}$ at (a) $T=300$, (b) 151 , and (c) $121 \mathrm{~K}$. Transitions from flicker noise dominance at low frequencies to white noise dominance at high frequencies are clearly observed. Although the slope of $S_{\text {Id }}(f)$ becomes very small at high frequencies, the slope at large $V_{\mathrm{d}}$ values is not quite zero. $S_{\text {Id }}(f)$ above $1 \mathrm{MHz}$ can be regarded as the sum of white noise and residual flicker noise. We therefore estimated the level of white noise, $S_{\mathrm{w}}$, and the corner frequency, $f_{\mathrm{c}}$, at which the intensity of flicker noise is equal to that of white noise, by using [18]

$$
S_{\text {Id }}(f)=K_{\mathrm{f}} / f^{\beta}+S_{\mathrm{w}}
$$
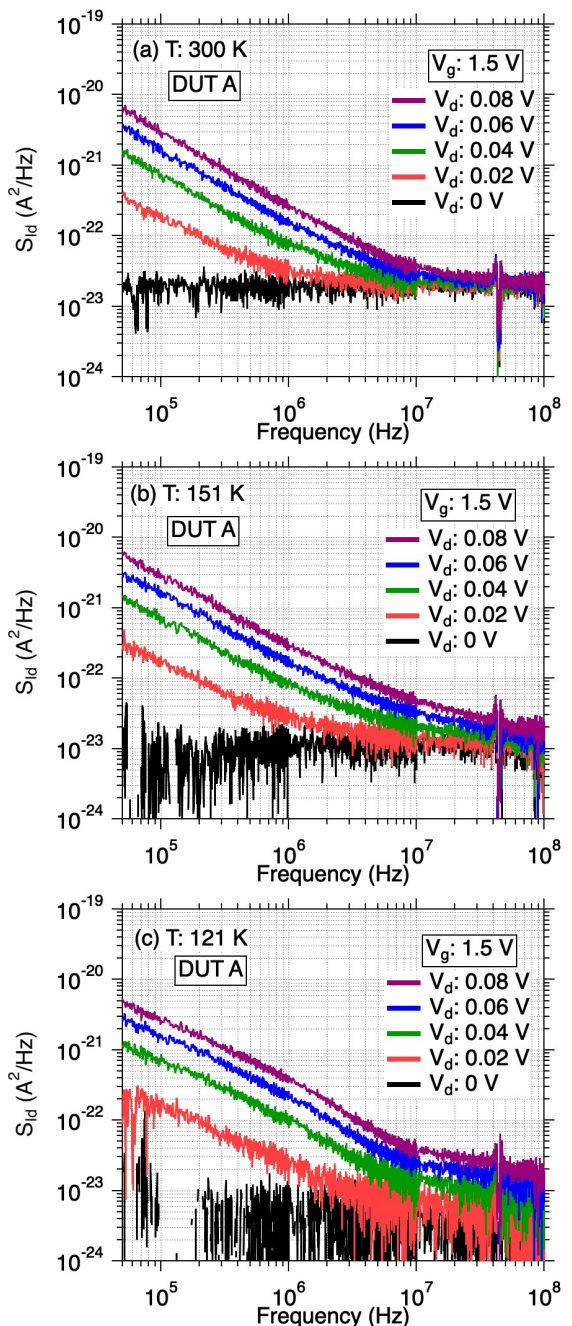

Fig. 10. Noise power spectral density $S_{\text {Id }}$ measured at (a) $T=300 \mathrm{~K}$, (b) $151 \mathrm{~K}$, and (c) $121 \mathrm{~K}$. 
where $K_{\mathrm{f}}$ is $S_{\text {Id }}$ at $1 \mathrm{~Hz}$, and $\left(K_{\mathrm{f}} / S_{\mathrm{w}}\right)^{1 / \beta}$ equals $f_{\mathrm{c}}$. Here, a value of $\beta$ averaged over $300 \mathrm{kHz}$ to $1 \mathrm{MHz}$ and $S_{\text {Id }}$ values at 10 and $30 \mathrm{MHz}$ at each $T$ were used to estimate $S_{\mathrm{w}}$ and $f_{\mathrm{c}}$. A straight $1 / f^{\beta}$ line on a $\log -\log S_{\text {Id }}$-versus- $f$ plot is assumed in (4). The accuracy of extraction of $S_{\mathrm{w}}$ by using (4) is affected by humps due to RTN. For example, this is observed as a broad hump in $S_{\text {Id }}$ (around $1 \mathrm{MHz}$ ) in Fig. 10(c). Hereafter, we will limit the lowest temperature analyzed by using (4) to $150 \mathrm{~K}$ because flicker noise below $150 \mathrm{~K}$ does not exhibit a good straight $1 / f^{\beta}$ line due to the appearance of RTN. As shown in Fig. 11, the corner frequency $f_{\mathrm{c}}$ gradually increases as $T$ decreases mainly because the intensity of thermal noise decreases. $f_{\mathrm{c}}$ is higher for larger $V_{\mathrm{d}}$ due to higher flicker noise intensity.

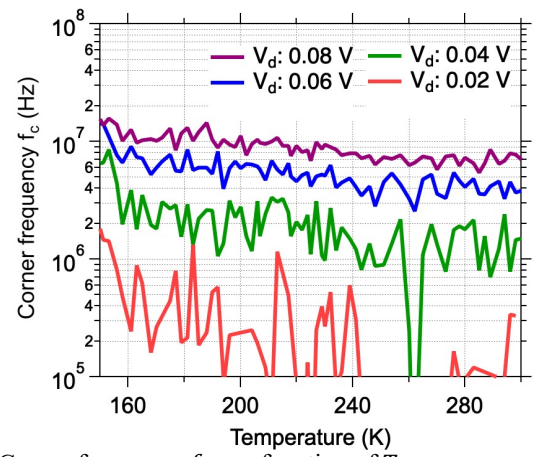

Fig. 11. Corner frequency $f_{\mathrm{c}}$ as a function of $T$.

Figure 12 shows the dependence of measured $S_{\text {Id }}$ and the estimated white noise $S_{\mathrm{w}}$ on $V_{\mathrm{d}}$ for (a) $T=300$ and (b) $151 \mathrm{~K}$. Open circles/squares and solid squares show measured $S_{\mathrm{Id}}$ at $10 / 30 \mathrm{MHz}$ and estimated $S_{\mathrm{w}}$ from (4), respectively. The difference between these values grows as $V_{\mathrm{d}}$ increases, resulting from the increase in residual flicker noise (Fig. 10). Thermal noise intensity $S_{\text {th }}$ for nonzero-drain-bias conditions is given by [40]

$$
S_{\mathrm{th}}=\frac{4 k_{\mathrm{B}} T R_{\mathrm{ch}}\left(V_{\mathrm{d}}\right)}{\left[R_{\mathrm{dif}}\left(V_{\mathrm{d}}\right)\right]^{2}}
$$

where $R_{\mathrm{ch}}=V_{\mathrm{d}} / I_{\mathrm{d}}$ is the chord resistance [41]. Note that $R_{\text {ch }}\left(V_{\mathrm{d}}\right) \neq R_{\text {dif }}\left(V_{\mathrm{d}}\right)$ unless $V_{\mathrm{d}}=0 \mathrm{~V}$.

Also plotted in Fig. 12 are the expected thermal noise $S_{\text {th }}$ (green dotted line) from (5), full shot noise $2 q I_{\mathrm{d}}$ (orange solid line), and the sum of these (blue dot-dashed line). At $V_{\mathrm{d}}=0 \mathrm{~V}$, $S_{\mathrm{w}}$ equals $S_{\mathrm{th}}$ and, as $V_{\mathrm{d}}$ increases, $S_{\mathrm{w}}$ increases along but below $2 q I_{\mathrm{d}}$ [18]. The latter gives the maximum possible value of shot noise intensity, realized when statistical fluctuation of the carrier number is at its maximum. Thermal noise $S_{\text {th }}$ dominates under low drain bias $\left(V_{\mathrm{d}} \lesssim 2 k_{\mathrm{B}} T / q\right)$ and shot noise becomes dominant when $V_{\mathrm{d}} \gtrsim 2 k_{\mathrm{B}} T / q$, as delineated by the red arrows. The values of $2 k_{\mathrm{B}} T / q$ for $T=300$ and $151 \mathrm{~K}$ are 52 and $26 \mathrm{mK}$, respectively. Therefore, as clearly observed in Fig. 12, the onset drain voltage of shot noise predominance at low temperature is smaller than that at room temperature.

Figure 13 shows the relation between the estimated white noise $S_{\mathrm{w}}$ and the thermal noise $S_{\mathrm{th}}$ at $150 \leq T \leq 300 \mathrm{~K}$. Although temperatures are not explicitly shown in Fig. 13,
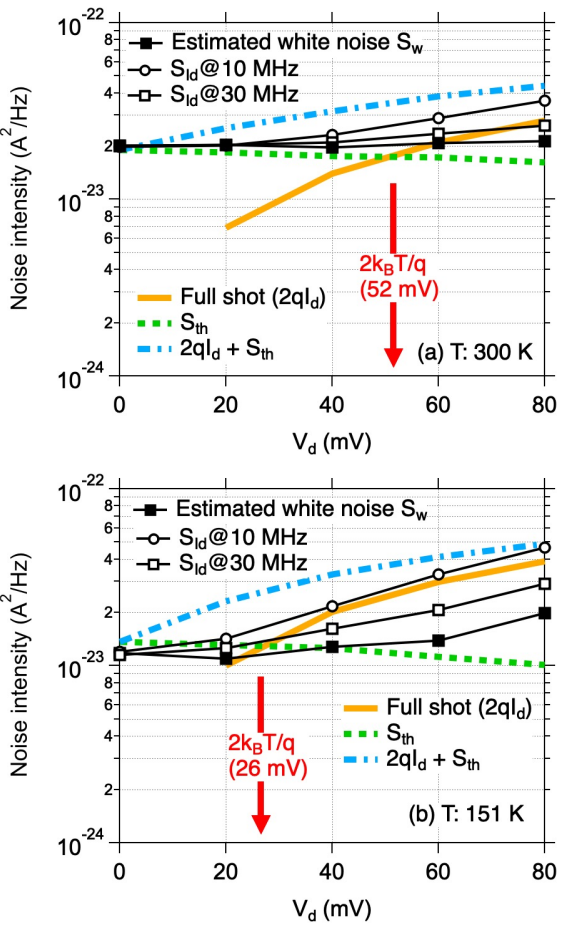

Fig. 12. Dependence of $S_{\text {Id }}$ extracted at 10 and $30 \mathrm{MHz}$, and estimated white noise $S_{\mathrm{w}}$ on $V_{\mathrm{d}}$ at $V_{\mathrm{g}}=1.5 \mathrm{~V}$ at (a) $T=300$ and (b) $151 \mathrm{~K}$.

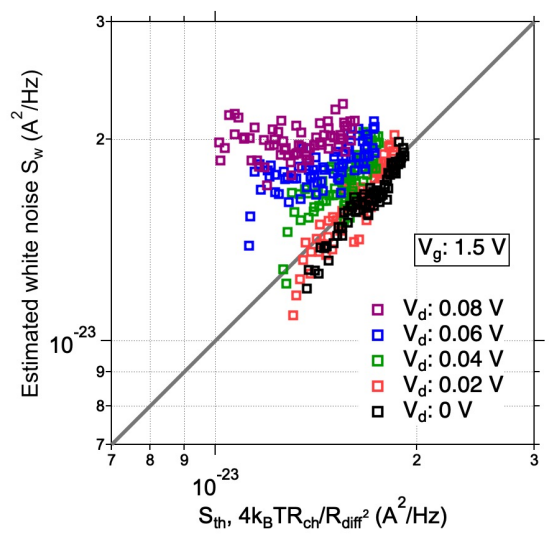

Fig. 13. Relation between the estimated white noise $S_{\mathrm{w}}$ and the thermal noise $S_{\text {th }}$ at $V_{\mathrm{d}}$ ranging from 0 to $0.08 \mathrm{~V}$.

decrease in the value of $S_{\text {th }}$ (horizontal axis) for each $V_{\mathrm{d}}$ corresponds to lowering of $T$. As $T$ decreases, $S_{\mathrm{w}}$ at $V_{\mathrm{d}}=0 \mathrm{~V}$ (black open squares) decreases, exhibiting a linear relationship with a nearly unity slope as was also seen in Fig. 9. In contrast, $S_{\mathrm{w}}$ (vertical axis) at $V_{\mathrm{d}}>0 \mathrm{~V}$ remains higher than the thermal noise $S_{\text {th }}$, clearly demonstrating that shot noise becomes predominant at low temperatures. Shot noise originates from the discrete nature of carrier transport. In MOSFETs, the shot noise is considered to originate primarily from carriers crossing the potential barrier near the source $[17,42]$, and secondarily from quasi-ballistic transport in the pinch-off region, where the inversion carrier density is low [43]. In this experiment, the observed shot noise should have resulted from the former because of the small values of $V_{\mathrm{d}}$. 
Shot noise is often characterized by using the so-called Fano factor $F(\leq 1)$ [44], given by the actual shot noise intensity divided by the full shot noise intensity, $2 q I_{\mathrm{d}}$. Successful use of $F$ for white noise modeling has been demonstrated for shortchannel MOSFETs [45]. A useful expression for measurementbased evaluation of $F$ applicable even near and at $V_{\mathrm{d}}=0 \mathrm{~V}$, where thermal noise and shot noise become indistinguishable, is [18]

$$
F=\frac{S_{\mathrm{w}}}{2 q I_{\mathrm{d}}+S_{\mathrm{th}}}
$$

Figure 14 shows temperature dependence of $F$ for $V_{\mathrm{d}}$ ranging from 0 to $0.08 \mathrm{~V}$. The black line in Fig. 14 is roughly equal to unity, which is the theoretically expected value of $F$ at $V_{\mathrm{d}}=0 \mathrm{~V}$. At room temperature, as $V_{\mathrm{d}}$ is raised from $0 \mathrm{~V}, F$ quickly falls in the linear region of MOSFET operation and becomes constant in the saturation region $[18,45]$. In this study, only the beginning of the linear region has been examined because of high $f_{\mathrm{c}}$ at larger values of $V_{\mathrm{d}}$ (Fig. 11). It is noteworthy that, in the linear region with $V_{\mathrm{d}}$ from 0.02 to $0.08 \mathrm{~V}, F$ slightly decreases as $T$ decreases (Fig. 14), and its $T$ dependence will presumably become weaker as $V_{\mathrm{d}}$ increases. However, further measurements at higher $V_{\mathrm{d}}$, including the saturation region [18], and at lower $T$ are definitely needed to be sure and for reliable cryogenic device modeling. Simulation with physical modeling, taking Coulomb interaction and the Pauli exclusion principle into consideration, will also be a powerful tool for further study $[43,46]$.

The lowest temperature for noise characterization in this study was limited to $120 \mathrm{~K}$ because bipolar transistors were adopted in the LNA. The measurement method proposed here should be applicable down to $4 \mathrm{~K}$ by using an LNA composed of MOSFETs or GaAs high electron mobility transistors (HEMTs).

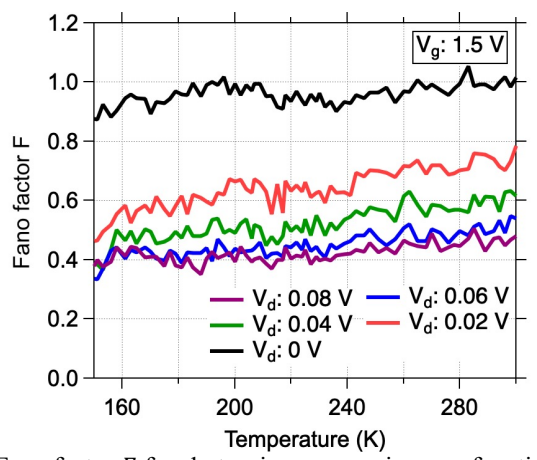

Fig. 14. Fano factor $F$ for shot-noise suppression as a function of $T$ at $V_{\mathrm{d}}$ ranging from 0 to $0.08 \mathrm{~V}$.

\section{CONCLUSIONS}

We have demonstrated variable-temperature characterization of N-MOSFETs, using the precisely calibrated PCB vehicle for broadband noise measurements from the flicker noise region to the white noise region. In order to build physics-based predictive models for MOSFETs, actual temperature-dependent measurements of broadband noise are necessary. Our measurement data show that a cryogenic environment reduces white noise at $V_{\mathrm{d}}=0 \mathrm{~V}$ as expected, but does not reduce shot noise that much at $V_{\mathrm{d}} \neq 0 \mathrm{~V}$. The latter will be a problematic noise source for applications in need of low-noise CMOS circuits.

\section{ACKNOWLEDGMENT}

The authors gratefully acknowledge the cooperative research with Prof. R. Hasunuma of University of Tsukuba.

\section{REFERENCES}

[1] S. Takagi, A. Toriumi, M. Iwase, and H. Tango, "On the universality of inversion layer mobility in Si MOSFET's: Part I-Effects of substrate impurity concentration," IEEE Trans. Electron Devices, vol. 41, no. 12, pp. 2357-2362, Dec. 1994.

[2] Y.-W. Yi, K. Masu, K. Tsubouchi, N. Mikoshiba, "Temperature-scaling theory for low-temperature-operated MOSFET with deep-submicron channel," Jpn. J. Appl. Phys., vol. 27, no. 10, p. L1958-L1961, Oct. 1988.

[3] H. L. Chiang, T. C. Chen, J. F. Wang, S. Mukhopadhyay, W. K. Lee, C. L. Chen, W. S. Khwa, B. Pulicherla, P. J. Liao, K. W. Su, K. F. Yu, T. Wang, H.-S. P. Wong, C. H. Diaz, J. Cai, "Cold CMOS as a powerperformance-reliability booster for advanced FinFETs," Symp. VLSI Technol., 2020, pp. 1-2, 10.1109/VLSITechnology18217.2020.9265065

[4] Y. Nakamura, Y. A. Pashkin, T. Yamamoto, and J. S. Tsai, "Charge echo in a Cooper-pair box," Phys. Rev. Lett., vol. 88, no. 4, p. 047901, Nov. 2016.

[5] Y. Nakamura, C. D. Chen, and J. S. Tsai, "Spectroscopy of energy-level splitting between two macroscopic quantum states of charge coherently superposed by Josephson coupling," Phys. Rev. Lett., vol. 117, no. 19, pp. 2328-2331, Jan. 2002.

[6] D. Sank, Z. Chen, M. Khezri, J. Kelly, R. Barends, B. Campbell, Y. Chen, B. Chiaro, A. Dunsworth, A. Fowler, E. Jeffrey, E. Lucero, A. Megrant, J. Mutus, M. Neeley, C. Neill, P. J. J. O’Malley, C. Quintana, P. Roushan, A. Vainsencher, T. White, J. Wenner, A. N. Korotkov, and J. M. Martinis, "Measurement-induced state transitions in a superconducting qubit: Beyond the rotating wave approximation," Phys. Rev. Lett., vol. 117, no. 19, 190503, Nov. 2016.

[7] P. Scarlino, D. J. van Woerkom, A. Stockklauser, J. V. Koski, M. C. Collodo, S. Gasparinetti, C. Reichl, W. Wegscheider, T. Ihn, K. Ensslin, and A. Wallraff, "All-microwave control and dispersive readout of gatedefined quantum dot qubits in circuit quantum electrodynamics," Phys. Rev. Lett., vol. 122, no. 20, 206802, May 2019.

[8] M. Mehrpoo, B. Patra, J. Gong, P. A. 't Hart, J. P. G. van Dijk, H. Homulle, G. Kiene, A. Vladimirescuy, F. Sebastiano, E. Charbon, M. Babaie, "Benefits and challenges of designing cryogenic CMOS RF circuits for quantum computers," International Symposium on Circuits and Systems, May 2019.

[9] F. Sebastiano, H. Homulle, B. Patra, R. Incandela, J. van Dijk, L. Song, M. Babaie, A. Vladimirescu, E. Charbon, "Cryo-CMOS electronic control for scalable quantum computing," 54th Annual Design Automation Conference, pp. 1-6, Jun. 2017.

[10] E. Charbon, M. Babaie, A. Vladimirescu, and F. Sebastiano, "Cryogenic CMOS circuits and systems-Challenges and opportunities in designing the electronic interface for quantum processors," IEEE Microwave Magazine. Electron Devices, vol. 22, no. 1, pp. 66-78, Jan. 2021.

[11] J. C. Bardin, D. H. Slichter, and D. J. Reilly, "Microwaves in quantum computing," IEEE J. Microwaves, vol. 1, no. 1, pp. 403-427, Jan. 2021.

[12] A. Beckers, F. Jazaeri, and C. Enz, "Theoretical limit of low Temperature subthreshold swing in field-effect transistors," IEEE. Electron Device Lett., vol. 41, no. 2, pp. 276-279, Feb. 2020.

[13] P. A. 't Hart, M. Babaie, E. Charbon, A. Vladimirescu, and F. Sebastiano,"Characterization and modeling of mismatch in cryoCMOS," J. Electron Device Society, vol. 8, pp. 263-273, Feb. 2020.

[14] A. Beckers, F. Jazaeri, and C. Enz, "Characterization and modeling of 28 -nm bulk CMOS technology down to $4.2 \mathrm{~K}$," J. Electron Device Society, vol. 6, pp. 1007-1018, Mar. 2018.

[15] R. Landauer, "Solid-state shot noise," Phys. Rev. B, vol. 47, pp. 16427 16432, June 1993.

[16] R. Sarpeshkar, T. Delbrück, and C. A. Mead, "White noise in MOS transistors and resistors," IEEE Circuits Devices Mag., vol. 9, no. 6, pp. 23-29, Nov. 1993. 
[17] R. Navid, C. Jungemann, T. H. Lee and R. W. Dutton, "High-frequency noise in nanoscale metal oxide semiconductor field effect transistors," $J$. Appl. Phys., vol. 101, no. 12, p. 124501, Jun. 2007.

[18] K. Ohmori and S. Amakawa, "Direct white noise characterization of short-channel MOSFETs," IEEE Trans. Electron Devices, vol. 68, no. 4, pp. 1478-1482, Apr. 2021.

[19] J. Chen, Z. S. He, D. Kuylenstierna, T. Eriksson, M. Hörberg, T Emanuelsson, T. Swahn, and H. Zirath, "Does LO noise floor limit performance in multi-gigabit millimeter-wave communication," IEEE Microwave Compon. Lett., vol. 27, no. 8, pp. 769-771, Aug. 2017.

[20] J. Chen, D. Kuylenstierna, S. E. Gunnarsson, Z. S. He, T. Eriksson, T Swahn, and H. Zirath, "Influence of white LO noise on wideband communication," IEEE Trans. Microwave Theory Tech., vol. 66, no. 7 , pp. 3349-3359, July 2018 .

[21] Keysight Technologies, "Fundamentals of RF and microwave noise figure measurements," Application Note, 5952-8255E, 2019.

[22] L. F. Tiemeijer, R. J. Havens, R. de Kort, and A. J. Scholten, "Improved Y-factor method for wide-band on-wafer noise-parameter measurements," IEEE Trans. Microwave Theory Tech., vol. 53, no. 9, pp. 2917-2925, Sep. 2005.

[23] A. Sheldon, L. Belostotski, H. Mani, C. E. Groppi, and K. F. Warnick, "Cryogenic noise-parameter measurements-Recent research and a fully automated measurement application," IEEE Microw. Mag., vol. 12, no. 8, pp. 52-64, Aug. 2021.

[24] R. Hu and S. Weinreb, "A novel wide-band noise-parameter measurement method and its cryogenic application," IEEE Trans. Microw. Theory Tech., vol. 52, no. 5, pp. 1498-1507, May 2004.

[25] K. Ohmori, R. Hasunuma, W. Feng, and K. Yamada, "Continuous characterization of MOSFET from low-frequency noise to thermal noise using a novel measurement system up to $100 \mathrm{MHz}$," Symp. VLSI Technol., 2012, pp. 143-144.

[26] K. Ohmori, R. Hasunuma, S. Yamamoto, Y. Tamura, H. Jiang, N. Ishihara, K. Masu, and K. Yamada, "Application of low-noise TIA ICs for novel sensing of MOSFET noise up to the GHz region," Symp. VLSI Circuits, 2013, pp. C40-41.

[27] Entrope Noise Probe, https://www.devicelab.co.jp

[28] K. Ohmori and S. Amakawa, "White noise characterization of NMOSFETs for physics-based cryogenic device modeling," Electron Devices Technology and Manufacturing Conference, 2021, pp. 1-3, doi: 10.1109/EDTM50988.2021.9420837.

[29] S. D. dos Santos, B. Cretu, V. Strobel, J.-M. Routoure, R. Carin, J.A. Martino, M. Aoulaiche, M. Jurczak, E. Simoen, C. Claey, "Lowfrequency noise assessment in advanced UTBOX SOI nMOSFETs with different gate dielectrics," Solid-State Electronics, vol. 97, pp. 14-22, Jul 2014.

[30] D. C. Murray, A. G. R. Evans, and J. C. Carter, "Shallow defects responsible for GR noise in MOSFET's," IEEE Trans. Electron Devices, vol. 38, no. 2, pp. 407-416, Feb. 1991.

[31] F. Yan, J. Bylander, S. Gustavsson, F. Yoshihara, K. Harrabi, D. G. Cory, T. P. Orlando, Y. Nakamura, J.-S. Tsai, and W. D. Oliver, "Spectroscopy of low-frequency noise and its temperature dependence in a superconducting qubit," Phys. Rev. Lett., vol. 85, no. 17, p. 174521, May 2012.

[32] H. Achour, B. Cretu, E. Simoen, J.-M. Routoure, R. Carin, A. Benfdila, M. Aoulaiche, and C. Claeys, "Identification of Si film traps in p-channel SOI FinFETs using low temperature noise spectroscopy," Solid-State Electron., vol 112, pp. 1-6, Oct. 2015.

[33] K. Ohmori, A. Shinoda, K. Kawai, Z. Wei, T. Mikawa, and R. Hasunuma, "Reduction of cycle-to-cycle variability in ReRAM by filamentary refresh," in Proc. Symp. VLSI Technol., 2017, pp. T90-91.

[34] Keysight Technologies, "Using noise floor extension in an X-series signal analyzer," Application Note, 5990-5340EN, 2019.

[35] G. Ghibaudo. O. Roux, C. Nguyen-Duc, F. Balestra, and J. Brini, "Improved analysis of low frequency noise in field-effect MOS transistors," Phys. Stat. Sol. (a), vol. 124, no. 2, pp. 571-581, Apr. 1991.

[36] L. K. J. Vandamme and F. N. Hooge, "What do we certainly know about $1 / f$ noise in MOSTs," IEEE Trans. Electron Devices, vol. 55, no. 11 pp. 3070-3085, Nov. 2008.

[37] R. Jayaraman and C. G. Sodini, "A $1 / f$ noise technique to extract the oxide trap density near the conduction band edge of silicon," IEEE Trans. Electron Devices, vol. 36, no. 9, pp. 1773-1782, Sep. 1989.

[38] M. J. Kirton and M. J. Uren, "Noise in solid-state microstructures: A new perspective on individual defects, interface states and low-frequency (1/f) noise," Advances in Physics, vol 38, no. 4, pp. 367-468, 1989.
[39] F. Yoshihara, K. Harrabi, A. O. Niskanen, Y. Nakamura, and J. S. Tsai, "Decoherence of flux qubits due to $1 / \mathrm{f}$ flux noise," Phys. Rev. Lett. vol. 97 , no. 16 , p. 167001 , Oct. 2006

[40] A. van der Ziel, Noise in Solid State Devices and Circuits. New York, NY, USA: John Wiley \& Sons, Inc., 1986.

[41] L. Chua, "Resistance switching memories are memristors," Appl. Phys. $A$, vol. 102, pp. 765-783, Mar. 2011.

[42] A. Rahman, J. Guo, S. Datta, and M. S. Lundstrom, "Theory of ballistic nanotransistors," IEEE Trans. Electron Devices, vol. 50, no. 9, pp. 1853 1864, Sep. 2003.

[43] N. Sano, K. Natori, K. Matsuzawa, and M. Mukai, "Current fluctuation characteristic of sub-0.1 micron device structures: a Monte Carlo study," Jpn. J. Appl. Phys., vol. 38, no. 5A, pp. L531-533, May 1999.

[44] U. Fano, "Ionization yield of radiations. II. The fluctuations of the number of ions," Phys. Rev., vol. 72, no. 1, pp. 26-29, July 1947.

[45] X. Chen, C. H. Chen, and R Lee, "Fast evaluation of the high-frequency channel noise in nanoscale MOSFETs," IEEE Trans. Electron Devices, vol. 65 , no. 4, pp. 1502-1509, Apr. 2018

[46] G. Iannaccone, A. Betti, G. Fiori, "Suppressed and enhanced shot noise in one dimensional field-effect transistors," J. Comput. Electron., vol. 14, pp. 94-104, Feb. 2015. 